Sharif University of Technology
Scientia Iranica
SCIENTIA

\title{
Forced vibration analysis of a system equipped with a Nonlinear Displacement-Dependent (NDD) damper
}

\author{
J. Jahanpour ${ }^{\mathrm{a}, *}$, M. Porghoveh ${ }^{\mathrm{a}}$ and S. Ilbeigi ${ }^{\mathrm{b}}$ \\ a. Department of Mechanical Engineering, Mashhad Branch, Islamic Azad University, Mashhad, Iran. \\ b. Department of Mechanical, Industrial and Systems Engineering, University of Rhode Island, Kingston, RI 02881, USA.
}

Received 14 October 2014; received in revised form 19 April 2015; accepted 9 May 2015

KEYWORDS
Nonlinear
Displacement-
Dependent (NDD)
damper;
Forced vibration
analysis;
Hard- and soft-
excitation;
Multiple scales
method;
Vibration reduction.

\section{Introduction}

Normally, to fully understand the vibration analysis of a vibratory system, two widely used types of vibration, namely, free vibration and forced vibration, must be investigated. In free vibration analysis, the system works without interaction with its environment, while, forced vibrations are excited by continuously applied, time-dependent external forces which do act on the system [1]. Most often, these are periodic forces. In many applications, vibration may cause discomfort, disturbance, and damage. In particular, in forced vi-

\footnotetext{
*. Corresponding author. Tel.: +985116625046, Ext. 445; Fax: +985116627560

E-mail addresses: jahanpourfr@mshdiau.ac.ir (J. Jahanpour); mdj.prg@hotmail.com (M. Porghoveh); ilbeigi@my.uri.edu (S. Ilbeigi)
}

\begin{abstract}
In this paper, the forced vibration analysis of a mass-spring system equipped soft- and hard- periodic excitations. In order to obtain the displacement of the excited several cases of hard- and soft-excitation and are also verified by the numerical fourth-order analyses. For the same external periodic force, a comparison has also been carried out better estimated response in the forced vibration analysis. Moreover, the NDD damper has

(C) 2016 Sharif University of Technology. All rights reserved.
\end{abstract}

bration, when the external excitation frequency reaches the natural frequency of the system, destruction of the system or the structure may occur $[2,3]$.

A general method to reduce the aforementioned undesired effects is adding a viscous damper to the vibratory system. Most viscous dampers have a constant damping coefficient. However, variable dampers have already found their way into industrial/commercial applications $[4,5]$. Variable dampers can be classified as active, semiactive and passive [6-10]. Active dampers are activated by an external source of power, which is usually provided by hydraulic actuators [1113]. In most cases, active dampers have high energy consumption, heavy weight, large size and high cost. Semiactive dampers inherit properties of controllable electromagnetic valves or Magneto Rheological (MR) fluid to control the damping characteristics of the system, and they are a compromise between active 
and passive dampers [5,14]. Passive dampers generally have fixed properties which are determined and preset according to the design goals and intended application [14]. Even though the active and semiactive dampers have higher performance, passive dampers are still the most commonly used [5].

While there are many types of passive damper, passive variable dampers have been recently developed. Among the passive variable dampers, those with stroke and displacement/position dependent sensitivity have been studied in several works [15-26]. Fukushima et al. [19] suggested that dampers should have a stroke dependent characteristic, such that for a given velocity, a longer stroke would give a greater force. However, the force in the stroke sensitivity cannot be identified directly with the actual position of the piston in the cylinder [4]. The displacement sensitive dampers have been used in aircraft landing gear, motorcycles, and vehicle suspension applications. The displacement sensitive schemes for landing gears employ positive recoil control or two and three level position dependent damping [20]. In a motorcycle front fork, a short and blunt rubber as a needle causes the damper orifice to close, which allows weaker springing with improved ride quality pressure [4]. Etman et al. [21] designed a stroke dependent damper for the front axle suspension of a truck. The displacement sensitive damper suggested for vehicle suspension applications follows the idea of using a long tapered needle entering an orifice in the piston [4,22]. This type of damper is merely limited to utilizing a tapered needle and is not mathematically modeled. Lee and Moon [23,24] reported on tests of a displacement sensitive damper with a longitudinally grooved pressure cylinder to relax the damping around the central position.

Some researchers have also thoroughly investigated nonlinear dampers [10,25-29]. For instance, Haque et al. [26] proposed an integral formulation to obtain the damping force of a displacement sensitive nonlinear damper. This method was based on transformation of the displacement sensitivity characteristic of the damper into a velocity sensitivity characteristic. Farjoud et al. [27] presented a nonlinear model of monotube hydraulic dampers with an emphasis on the effects of shim stack on damper performance. Guo et al. [28] studied the force and displacement transmissibility of nonlinear viscous damper based vibration isolation.

Free and forced vibration analyses of systems equipped with nonlinear damper have also been studied in several pieces of research [29-33]. For instance, Bugra et al. [31] implemented several experiments to determine the dynamic characterization of nonlinear oil-free wire mesh dampers. To this end, free and forced vibrations were investigated using the Hilbert transform procedure and controlled amplitude single frequency excitation tests, respectively. Main and Jones [32] demonstrated the free vibration of a taut cable with a nonlinear amplitude dependent damper. Diotallevi et al. [33] proposed a simplified design method to analyze the forced vibration of Single- and Multi-Degrees of Freedom (SDF and MDF) systems equipped with nonlinear viscous dampers under harmonic external forces. In their work, the responses of the SDF and MDF systems were also calculated numerically.

Recently, Ilbeigi et al. [29] introduced a novel scheme for Nonlinear Displacement-Dependent (NDD) dampers in which the damping coefficient and damping force were both continuous and smooth functions of displacement. In contrast with a linear damper, where the damping force only depends on the velocity, the damping force produced by the proposed NDD damper depends on the position of the system, as well as the velocity. In their work, a vibratory mass-spring system equipped with a NDD damper was also mathematically modeled and free vibration analysis of the system was undertaken. The results presented in [29] indicate that the advised passive NDD damper scheme is capable of reducing the free vibration amplitude rather better than the existing traditional linear damper.

In this paper, the nonlinear differential equation of a mass-spring system utilizing the NDD damper with an external force is derived. To study the forced vibration analysis of the system, the external excitation has been categorized into two types; hard and soft, and the response of the excited system for each type is elaborated upon using the multiple scales method.

The rest of the paper is organized as follows. Section 2 is a review on the NDD damper mechanism. In Section 3, the mathematical model of the nonlinear mass-spring system, equipped with the NDD damper, with an external force, is formulated, and the governing differential equation is derived. The forced vibration analysis of the system for two type of soft- and hardexcitation is also illustrated in this section. Several numerical examples are presented in Section 4. The results are analyzed and discussed in Section 5. Finally, Section 6 concludes the paper.

\section{Review of NDD damper mechanism}

Most viscous dampers have a piston with one or more orifices on their body. This piston moves inside a cylinder filled with a viscous fluid (Figure 1) [29], and the viscosity of the fluid results in a resistant force along the movement. This damping force is linearly related to the velocity of the piston as follows:

$$
F=-c \frac{d u}{d t},
$$

in which $c$ is the damping coefficient. 


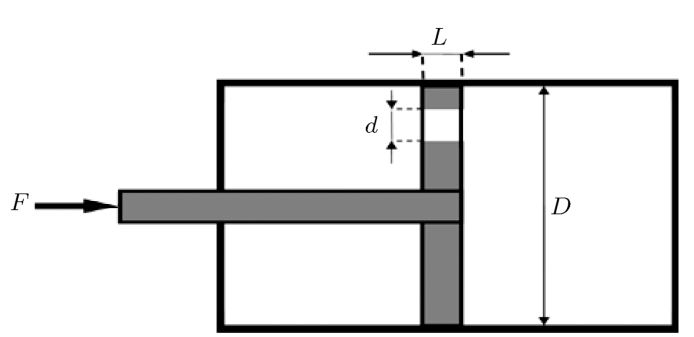

Figure 1. Schematic of a simple viscous damper.

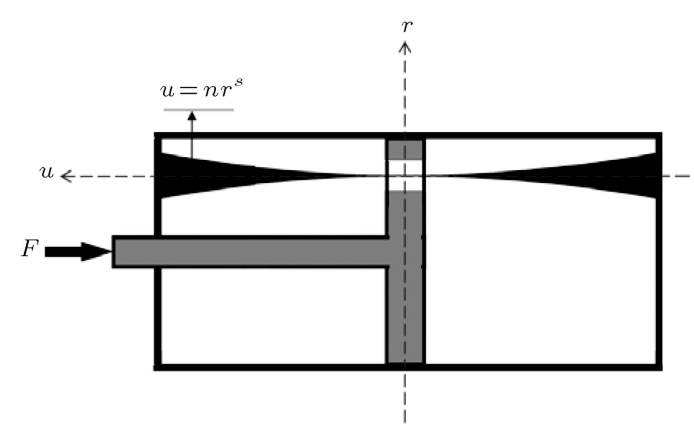

Figure 2. Schematic of the designed nonlinear displacement-dependent damper [29].

To obtain the value of the damping coefficient, one can use the Hagen-Poiseuille equation for laminar flows. Assuming there is only one orifice in the piston, $c$ can be obtained by the following equation:

$$
c_{\text {linear }}=\hbar\left[\left(\frac{D}{d}\right)^{2}-1\right]^{2},
$$

where, $D$ and $d$ are the cylinder diameter and the orifice diameter on the piston, respectively. Also, $\hbar=8 \pi \mu L$ in which $L$ is the piston thickness and $\mu$ indicates the dynamical viscosity of the fluid used in the damper.

Should parameters $D, d$ and $L$ be constant variable, the damping coefficient will be, therefore, a constant value. In order to modify the current form of the damper and create one giving better performance, [29] placed a solid cone with variable diameter along the $u$-axis in the cylinder (see Figure 2 ). In their work, the cone diameter was considered to be a function of the piston position as follows:

$$
u=n r^{s} \quad \text { or } \quad r=\left(\frac{u}{n}\right)^{\frac{1}{s}} .
$$

Hence, in the new damper, the orifice diameter becomes equal to $d-2 r(u)$. Substituting $d-2 r$ for $d$, and $r$ from Eq. (3) in Eq. (2) leads to:

$$
c=\hbar\left[\gamma^{2}\left(\frac{1}{1-\beta u^{\left(\frac{1}{s}\right)}}\right)^{2}-1\right]^{2}
$$

where, $\beta=\frac{2}{d . n\left(\frac{1}{s}\right)}$ and $\gamma=\frac{D}{d}$.

Applying the 2nd order Taylor series expansion,
Eq. (4) can be expressed as follows [29]:

$$
c=\alpha_{1}+\alpha_{2}|u|^{\left(\frac{1}{s}\right)}+\alpha_{3}|u|^{\left(\frac{2}{s}\right)}+\alpha_{4}|u|^{\left(\frac{3}{s}\right)}+\alpha_{5}|u|^{\left(\frac{4}{s}\right)} .
$$

Supposing $n=1$ and $s=\frac{1}{2}$, Eq. (5) can be rewritten as follows:

$$
c=\alpha_{1}+\alpha_{2} u^{2}+\alpha_{3} u^{4}+\alpha_{4} u^{6}+\alpha_{5} u^{8} .
$$

Parameters, $\alpha_{i}$, in Eq. (6), and further details of the mathematical formulation of the NDD damper can be found in [29].

\section{Forced vibration analysis of a mass-spring-NDD damper system}

The governing differential equation of a simple massspring-damper system with an external periodic force is as follows:

$$
\ddot{u}+\frac{c}{m} \dot{u}+\omega_{n}^{2} u=\frac{F \cos (\Omega t)}{m},
$$

in which, $\frac{c}{m}=2 \zeta \omega_{n}$. Also, $\omega_{n}=\sqrt{\frac{k_{s}}{m}}$ and $\Omega$ are the natural frequency and excitation frequency of the system, respectively.

For a linear damper with the damping ratio of $\zeta<1$, the solution of Eq. (7) can be expressed in the following form [2]:

$$
\begin{aligned}
u_{l}(t)= & A_{1} e^{-\zeta \omega_{n} t} \sin \left(\sqrt{1-\zeta^{2}} \omega_{n} t+\psi\right) \\
& +A_{2} \cos (\Omega t-\varphi),
\end{aligned}
$$

where, $A_{1}$ and $\psi$ are evaluated using initial conditions. Also, $A_{2}$ and $\varphi$ are computed as:

$$
\begin{aligned}
& A_{2}=\frac{\frac{F}{k_{s}}}{\sqrt{\left(1-\left(\frac{\Omega}{\omega_{n}}\right)^{2}\right)^{2}+\left(2 \zeta \frac{\Omega}{\omega_{n}}\right)^{2}}}, \\
& \varphi=\tan ^{-1}\left(\frac{2 \zeta \frac{\Omega}{\omega_{n}}}{1-\left(\frac{\Omega}{\omega_{n}}\right)^{2}}\right) .
\end{aligned}
$$

The damping force is calculated by the following equation:

$$
F_{\text {Damping }}=c \frac{d u(t)}{d t} .
$$

To achieve the governing differential equation of a basic mass-spring system equipped with a NDD damper bearing an external periodic excitation, $c$ from Eq. (6) must be replaced into Eq. (7), as follows: 


$$
\begin{aligned}
\frac{d^{2} u}{d \hat{t}^{2}}+u= & -\frac{\hbar\left(\gamma^{4}-2 \gamma^{2}+1\right)}{\sqrt{m k_{s}}}\left(1+\beta_{1} u^{2}+\beta_{2} u^{4}\right. \\
& \left.+\beta_{3} u^{6}+\beta_{4} u^{8}\right) \frac{d u}{d \hat{t}}+\frac{F}{k_{s}} \cos (\lambda \hat{t})
\end{aligned}
$$

Or:

$$
\begin{aligned}
\frac{d^{2} u}{d \hat{t}^{2}}+u= & -\varepsilon\left(1+\beta_{1} u^{2}+\beta_{2} u^{4}+\beta_{3} u^{6}+\beta_{4} u^{8}\right) \frac{d u}{d \hat{t}} \\
& +\frac{F}{k_{s}} \cos (\lambda \hat{t})
\end{aligned}
$$

in which, $\hat{t}=\omega_{n} t, \varepsilon=\frac{\hbar\left(\gamma^{4}-2 \gamma^{2}+1\right)}{\sqrt{m k_{s}}}$, and $\lambda=\frac{\Omega}{\omega_{n}}$. Also, $\beta_{i}=\frac{\alpha_{i+1}}{\alpha_{1}}$ are given in Appendix A.

\subsection{A brief discussion on parameter $\varepsilon$}

As can be seen in Eqs. (11), the terms in parenthesis cause the governing differential equation to be nonlinear. $\varepsilon$ defines their nonlinear effect on the problem; should $\varepsilon$ have a substantial value, the governing equation is categorized as a strongly nonlinear problem, and if $\varepsilon$ has a small value, the governing equation is characterized as a weakly nonlinear problem. It should be noted that the procedure of obtaining the analytical solution for each category differs considerably. Thus, identifying the coefficient determining the strength of the nonlinear terms, i.e. $\varepsilon$ in this paper, based on the system parameters, must be the first step taken in order to understand the nature of the nonlinearity.

In this study $\varepsilon=\frac{\hbar\left(\gamma^{4}-2 \gamma^{2}+1\right)}{\sqrt{m k_{s}}}$. So, it is independent of the shape parameters $n$ and $s$. But, it is directly proportional to $\hbar$, and, accordingly, to the fluid viscosity. It is also dependent on $\gamma$. Hence, increasing the viscosity or $\hbar$, causes increasing $\varepsilon$, and strengthens the nonlinear effects of the terms in parenthesis in Eq. (11), successively. In addition, increasing $k_{s}$ or $m$ would cause decreasing $\varepsilon$ and a decrease in the strength of nonlinearity in Eq. (11) as well.

Various perturbation methods are available for solving weakly nonlinear differential equations. In other words, for small values of $\varepsilon$, perturbation techniques are well capable of obtaining a precise solution. Therefore, in this study, the parameters upon which $\varepsilon$ depends are chosen in such a way that $\varepsilon$ has small values.

In most nonlinear governing differential equations of nonlinear dynamic systems, there is a parameter, such as $\varepsilon$ in this study, which describes the strength of the nonlinear terms in the equation. However, it is worth mentioning that in each nonlinear system, $\varepsilon$ is reliant on the characteristics of that system. Hence, if one wants to use the perturbation method and obtain accurate results, all the parameters affecting the coefficient of the nonlinear terms should be exactly discovered for each system. It is worth mentioning that assuming the value of $\varepsilon$ to be small, arbitrarily, without any identification of the effecting parameters cannot lead to a precise solution, because the value of $\varepsilon$ should be defined according to system parameters.

The shape parameters, i.e. $n$ and $s$, have an effect on $\beta$, as $\beta=\frac{2}{d \cdot n^{\frac{1}{s}}}$, and, successively, on the damping coefficient, $c$, as given by Eq. (4). Accordingly, the governing equation of the vibration system utilizing the NDD damper with an external periodic excitation is affected by these shape parameters. Since the central focus of this study is to analyze the forced vibration of a mass-spring system equipped with a NDD damper, in this paper, the shape parameters have been selected as a fixed set. Nevertheless, a couple of the values of these parameters can be optimized, according to the design goal and intended particular application.

To analyze the forced vibration of a mass-spring system equipped with a NDD damper, four categories arise depending on whether the external excitation force is small titled as "soft", or large titled as "hard", and whether the excitation is resonant, i.e. $\lambda-\omega_{n}=$ $O(\varepsilon)$, or non-resonant, i.e. $\lambda-\omega_{n}=O(1)$. In this paper, we consider two types of external excitation; soft-non-resonant and hard-non-resonant.

In the following subsection, the procedure of employing the Multiple Scales Method (MSM) as a perturbation technique is illustrated to solve Eq. (11) for the two aforementioned forced vibration types, i.e. soft- and hard-non-resonant excitations.

\subsection{Soft-non-resonant excitation analysis using $M S M$}

The Multiple Scales Method (MSM) is based on the idea of representing multiple independent variables as functions of the time variable and expressing all other time dependent functions as functions of represented variables $[34,35]$. For this aim, the independent variables are introduced as:

$$
T_{n}=\varepsilon^{n} \hat{t} \quad \text { for } \quad n=0,1,2 \cdots
$$

Any time dependent function, including the solution of the governing equation of a nonlinear system, is represented as:

$$
\begin{aligned}
u= & u_{0}\left(T_{0}, T_{1}, T_{2}\right)+\varepsilon u_{1}\left(T_{0}, T_{1}, T_{2}\right)+\varepsilon^{2} u_{2}\left(T_{0}, T_{1}, T_{2}\right) \\
& +O\left(\varepsilon^{3}\right) .
\end{aligned}
$$

With regard to the chain rule of derivation, the first and second derivatives, with respect to $\hat{t}$, can be expressed in the following forms:

$$
\begin{aligned}
& \frac{d}{d \hat{t}}=D_{0}+\varepsilon D_{1}+\varepsilon^{2} D_{2}, \\
& \frac{d^{2}}{d \hat{t}^{2}}=D_{0}^{2}+2 \varepsilon D_{0} D_{1}+\varepsilon^{2}\left(D_{1}^{2}+2 D_{0} D_{2}\right),
\end{aligned}
$$

where $D_{n}=\frac{\partial}{\partial T_{n}}$. 
According to [35], to analyze the soft-nonresonant excitation of the system, as the amplitude of external force is small, the amplitude must be considered to be in the order of $\varepsilon$. Also, increasing the external force value would decrease the accuracy of the solution. The related external force term in Eqs. (11) can be considered as follows:

$$
\frac{F}{k_{s}} \cos (\lambda \hat{t})=\varepsilon k \cos (\lambda \hat{t})
$$

where, $k$ is the coefficient related to the external force amplitude, and $k=O(1)$ [35]. In this study, $k$ has been selected to be between 3 and 30 , as it is 3 for $\varepsilon=0.5$ and 30 for $\varepsilon=0.05$. Therefore, Eq. (11) can be rewritten as follows:

$$
\begin{aligned}
\frac{d^{2} u}{d \hat{t}^{2}}+u= & -\varepsilon\left(1+\beta_{1} u^{2}+\beta_{2} u^{4}+\beta_{3} u^{6}+\beta_{4} u^{8}\right) \frac{d u}{d \hat{t}} \\
& +\varepsilon k \cos (\lambda \hat{t}) .
\end{aligned}
$$

Substituting Eqs. (13) and (14) into Eq. (16) and then equating the coefficient of each power of $\varepsilon$ in both sides of Eq. (16) together, leads to:

$$
\begin{aligned}
D_{0}^{2} u_{0}+u_{0}= & 0, \\
D_{0}^{2} u_{1}+u_{1}= & -2 D_{0} D_{1} u_{0}-\left(1+\beta_{1} u_{0}^{2}+\beta_{2} u_{0}^{4}\right. \\
& \left.+\beta_{3} u_{0}^{6}+\beta_{4} u_{0}^{8}\right) D_{0} u_{0}+k \cos \left(\lambda T_{0}\right), \\
D_{0}^{2} u_{2}+u_{2}= & -2 D_{0} D_{1} u_{1}-2 D_{0} D_{2} u_{0}-D_{1}^{2} u_{0} \\
- & \left(1+\beta_{1} u_{0}^{2}+\beta_{2} u_{0}^{4}+\beta_{3} u_{0}^{6}+\beta_{4} u_{0}^{8}\right) D_{1} u_{0} \\
& -\left(1+\beta_{1} u_{0}^{2}+\beta_{2} u_{0}^{4}+\beta_{3} u_{0}^{6}+\beta_{4} u_{0}^{8}\right) D_{0} u_{0} \\
& -\left(2 \beta_{1} u_{0} u_{1}+4 \beta_{2} u_{0}^{3} u_{1}+6 \beta_{3} u_{0}^{5} u_{1}\right) D_{0} u_{0} .
\end{aligned}
$$

Eq. (17) is a homogenous linear partial differential equation. The general solution of Eq. (17) can be written as:

$$
u_{0}=A\left(T_{1}, T_{2}\right) e^{i T_{0}}+\bar{A}\left(T_{1}, T_{2}\right) e^{-i T_{0}} .
$$

It is observed that term $u_{0}$ of the response of the system is independent of the external force (see Eqs. (17) and $(20))$.

Substituting $u_{0}$ from Eq. (20) into Eq. (18) leads to:

$$
\begin{array}{r}
D_{0}^{2} u_{1}+u_{1}=-i\left[\Delta_{1} e^{i T_{0}}+\frac{i k}{2} e^{i \lambda T_{0}}+\Delta_{2} e^{3 i T_{0}}\right. \\
\left.+\Delta_{3} e^{5 i T_{0}}+\Delta_{4} e^{7 i T_{0}}+\Delta_{5} e^{9 i T_{0}}+C C\right],
\end{array}
$$

where, $\Delta_{i}^{\prime} s$ are given in Appendix $\mathrm{A}$ and $C C$ is a symbol for complex conjugate.

In order to achieve first order approximation, the secular term must be omitted from Eq. (21), and, therefore, the coefficients of $e^{ \pm i T_{0}}$ must be vanished; that is:

$$
\Delta_{1}=0 \text {. }
$$

Having eliminated the secular terms from Eq. (21) and considering its general homogenous response as:

$$
B\left(T_{1}, T_{2}\right) e^{i T_{0}}+\bar{B}\left(T_{1}, T_{2}\right) e^{i T_{0}},
$$

the general solution of Eq. (21) can be expressed in the following form:

$$
\begin{aligned}
u_{1}= & B\left(T_{1}, T_{2}\right) e^{i T_{0}}+\frac{k}{2\left(1-\lambda^{2}\right)} e^{i \lambda T_{0}}+\frac{1}{8} i \Delta_{2} e^{3 i T_{0}} \\
& +\frac{1}{24} i \Delta_{3} e^{5 i T_{0}}+\frac{1}{48} i \Delta_{4} e^{7 i T_{0}}+\frac{1}{80} i \Delta_{5} e^{9 i T_{0}}+C C .
\end{aligned}
$$

Also, term $\frac{k}{2\left(1-\lambda^{2}\right)} e^{i \lambda T_{0}}$ is the effect of the external periodic force on term $u_{1}$ of the response of the system.

In order to solve Eq. (22), the unknown function, $A$, is considered in its most general form as follows:

$$
A=\frac{1}{2} a\left(T_{1}, T_{2}\right) e^{i \phi\left(T_{1}, T_{2}\right)},
$$

where, $a\left(T_{1}, T_{2}\right)$ mainly describes the response's magnitude of the system, which is a small value for all $T_{1}=\varepsilon \hat{t}$ and $T_{2}=\varepsilon^{2} \hat{t}$.

Substituting $A$ from Eq. (24), and its conjugate and derivative into Eq. (22) leads to:

$$
-\left(\frac{\partial a}{\partial T_{1}}+i a \frac{\partial \phi}{\partial T_{1}}\right)=c_{1} a+c_{3} a^{3}+c_{5} a^{5}+c_{7} a^{7}+c_{9} a^{9}
$$

where $c_{i}^{\prime} s$ are given in Appendix A, and are affected neither by the excitation amplitude nor the excitation frequency. According to the fact that $c_{i}^{\prime} s$ are real numbers, separating the real and imaginary parts in Eq. (25) results in:

$$
\begin{aligned}
\frac{\partial \phi}{\partial T_{1}} & =0 \\
\frac{\partial a}{\partial T_{1}} & =c_{1} a+c_{3} a^{3}+c_{5} a^{5}+c_{7} a^{7}+c_{9} a^{9} .
\end{aligned}
$$

Hence, $\phi=\phi\left(T_{2}\right)$. Besides, $a\left(T_{1}, T_{2}\right)$ can be solved by integration from Eq. (26b). Because the magnitude of the system response is small, terms $a^{5}, a^{7}$ and $a^{9}$ can be neglected, and, therefore, the integration from Eq. (26b) leads to:

$$
a\left(T_{1}, T_{2}\right)=\sqrt{\frac{c_{1} a_{0}^{2}}{\left(c_{1}+c_{3} a_{0}^{2}\right) e^{2 c_{1} T_{1}}-c_{3} a_{0}^{2}}}+g\left(T_{2}\right),
$$


in which, $a_{0}$ is calculated by applying the initial conditions. $g\left(T_{2}\right)$ is also obtained from the following procedure to determine the second-order approximation for $u$.

To determine the second-order approximation for $u ; u_{0}$ and $u_{1}$, from Eqs. (20) and (23), must be substituted into Eq. (19) as follows:

$$
D_{0}^{2} u_{2}+u_{2}=Q\left(T_{1}, T_{2}\right) e^{i T_{0}}+\bar{Q}\left(T_{1}, T_{2}\right) e^{-i T_{0}}+N S T,
$$

where, NST stands for non-secular terms, and:

$$
\begin{aligned}
& Q\left(T_{1}, T_{2}\right)=-i\left(B+\beta_{1} A^{2} \bar{B}+2 \beta_{1} A \bar{A} B\right. \\
& \quad+4 \beta_{2} A^{3} \bar{A} \bar{B}+6 \beta_{2} A^{2} \bar{A}^{2} B+15 \beta_{3} A^{4} \bar{A}^{2} \bar{B} \\
& \left.\quad+20 \beta_{4} A^{3} \bar{A}^{3} B+56 \beta_{4} A^{5} \bar{A}^{3} \bar{B}+70 \beta_{4} A^{4} \bar{A}^{4} B\right) \\
& \quad-D_{1}^{2} A-2 i D_{1} B-2 i D_{2} A-\left(1+2 \beta_{1} A \bar{A}\right. \\
& \left.\quad+6 \beta_{2} A^{2} \bar{A}^{2}+20 \beta_{3} A^{3} \bar{A}^{3}+70 \beta_{4} A^{4} \bar{A}^{4}\right) D_{1} A \\
& \quad-\left(\beta_{1} A^{2}+4 \beta_{2} A^{3} \bar{A}+15 \beta_{3} A^{4} \bar{A}\right. \\
& \left.\quad+56 \beta_{4} A^{5} \bar{A}^{3}\right) D_{1} \bar{A}+q\left(T_{1}, T_{2}\right)
\end{aligned}
$$

in which, $q\left(T_{1}, T_{2}\right)$ is given in Appendix A.

Term $\frac{k}{2\left(1-\lambda^{2}\right)} e^{i \lambda T_{0}}$, which is related to the external periodic force, has only an effect on the non-secular terms. That is, the secular terms are independent of the external force. Secular terms will be omitted if $Q=0$. To solve Eq. (28b) with $Q=0$, one let;

$$
B=\frac{1}{2} i b e^{i \phi} .
$$

Substituting for $A$ and $B$ from Eqs. (24) and (29) into Eq. (28b), with $Q=0$, and separating the real and imaginary parts, yields the following relations to obtain $g\left(T_{2}\right), b$ and $\phi[29]$ :

$$
\begin{aligned}
& \left\{\begin{array}{l}
\int d\left(\frac{b}{a}\right)=-\left(\frac{d \phi}{d T_{2}}+\frac{1}{16}\right) T_{1}+\int\left(\frac{1}{8 a}+\frac{7}{32} \beta_{1} a\right) d a \\
\frac{\partial a}{\partial T_{2}}=0 \rightarrow a=a\left(T_{1}\right)
\end{array}\right. \\
& \phi=-\frac{1}{16} T_{2}+\phi_{0}
\end{aligned}
$$

where $\phi_{0}$ is a constant value. In addition, according to Eqs. (27) and (30), $g\left(T_{2}\right)$ must be equal to a constant value, which can be considered to be zero. Thus:

$$
a(t)=\sqrt{\frac{c_{1} a_{0}^{2}}{\left(c_{1}+c_{3} a_{0}^{2}\right) e^{2 c_{1} \varepsilon \omega_{n} t}-c_{3} a_{0}^{2}}} .
$$

As a result, to an error of $O\left(\varepsilon^{2}\right)$ and substituting $u_{0}$ and $u_{1}$ into Eq. (13), the expansion of $u(t)$ to the second approximation is:

$$
\begin{aligned}
& u(t)=\sqrt{\frac{c_{1} a_{0}^{2}}{\left(c_{1}+c_{3} a_{0}^{2}\right) e^{2 c_{1} \varepsilon \omega_{n} t}-c_{3} a_{0}^{2}}} \cos \\
& {\left[\omega_{n} t\left(1-\frac{1}{16} \varepsilon^{2}\right)+\phi_{0}\right]+\frac{F}{k_{s}\left(1-\lambda^{2}\right)} \cos (\Omega t)} \\
& -\varepsilon\left\{\frac{1}{4} \Upsilon_{1} \sin 3\left[\omega_{n} t\left(1-\frac{1}{16} \varepsilon^{2}\right)+\phi_{0}\right]\right. \\
& +\frac{1}{12} \Upsilon_{2} \sin 5\left[\omega_{n} t\left(1-\frac{1}{16} \varepsilon^{2}\right)+\phi_{0}\right] \\
& +\frac{1}{24} \Upsilon_{3} \sin 7\left[\omega_{n} t\left(1-\frac{1}{16} \varepsilon^{2}\right)+\phi_{0}\right] \\
& \left.+\frac{1}{40} \Upsilon_{4} \sin 9\left[\omega_{n} t\left(1-\frac{1}{16} \varepsilon^{2}\right)+\phi_{0}\right]\right\}+O\left(\varepsilon^{2}\right),
\end{aligned}
$$

where $\Upsilon_{i}^{\prime}$ s are given in Appendix A. $a_{0}=\left.a(\hat{t})\right|_{\hat{t}=0}$, and $\phi_{0}$ can be calculated by applying the initial conditions of the system.

It should be noted that the $\varepsilon^{2}$ in Eq. (33) is the effect of $T_{2}=\varepsilon^{2} \hat{t}$ via Eq. (31) on the frequency of response of the system, and the response of the system is still a time dependent function with the accuracy of the $O\left(\varepsilon^{2}\right)$. Parameters $c_{1}, c_{3}, c_{5}, c_{7}$ and $c_{9}$; and also $\Upsilon_{1}, \Upsilon_{2}, \Upsilon_{3}$, and $\Upsilon_{4}$ in the second approximate solution for the soft-non-resonant excited system via Eq. (33), are dependent on $\varepsilon, \beta_{1}, \beta_{2}, \beta_{3}$ and $\beta_{4}$. Consequently, they are also dependent on system characteristics such as spring stiffness, mass, dynamic viscosity, orifice diameter and piston width.

\subsection{Hard-non-resonant excitation analysis using $M S M$}

For analyzing the hard-non-resonant excitation of the system, the amplitude of the periodic force, i.e. $F$, in Eq. (11) is not small.

Time scales for applying the multiple scales method to solve Eq. (11) are chosen as follows:

$$
T_{0}=\hat{t}, \quad T_{1}=\varepsilon \hat{t}
$$

The approximate solution of Eq. (11) can be represented in the form of:

$$
u=u_{0}\left(T_{0}, T_{1}\right)+\varepsilon u_{1}\left(T_{0}, T_{1}\right)+O\left(\varepsilon^{2}\right) .
$$

With regard to the chain rule of derivation, the firstand second derivatives, with respect to $\hat{t}$, can be represented as: 


$$
\begin{aligned}
& \frac{d}{d \hat{t}}=D_{0}+\varepsilon D_{1}, \\
& \frac{d^{2}}{d \hat{t}^{2}}=D_{0}^{2}+2 \varepsilon D_{0} D_{1}+\varepsilon^{2} D_{1}^{2} .
\end{aligned}
$$

Substituting Eqs. (35) and (36) into Eq. (11), and equating the coefficient of each power of $\varepsilon$ in both sides of Eq. (11) with each other, leads to:

$$
\begin{aligned}
D_{0}^{2} u_{0}+u_{0}= & \frac{F}{k_{s}} \cos \left(\lambda T_{0}\right), \\
D_{0}^{2} u_{1}+u_{1}= & -2 D_{0} D_{1} u_{0}-\left(1+\beta_{1} u_{0}^{2}+\beta_{2} u_{0}^{4}\right. \\
& \left.+\beta_{3} u_{0}^{6}+\beta_{4} u_{0}^{8}\right) D_{0} u_{0} .
\end{aligned}
$$

In contrast to soft-excitation analysis, in which the coefficient related to the external force amplitude does not have any effect on $u_{0}$, for the type of hardexcitation, the external force appears in the equation containing $u_{0}$ (see Eq. (37)). Thus, in this case, according to Eqs. (37) and (38), the external force affects $u_{0}$, and, consequently, $u_{1}$.

The general solution of Eq. (37) can be expressed as:

$$
\begin{aligned}
u_{0}= & A_{H}\left(T_{1}\right) e^{i T_{0}}+\bar{A}_{H}\left(T_{1}\right) e^{i T_{0}} \\
& +\frac{F}{k_{s}\left(1-\lambda^{2}\right)} \cos \left(\lambda T_{0}\right) .
\end{aligned}
$$

The influence of external force on the term, $u_{0}$, of the response of the system is clearly seen in Eq. (39).

Substituting for $u_{0}$ from Eq. (39) into Eq. (38) results in:

$$
\begin{aligned}
D_{0}^{2} u_{1} & +u_{1}=-i\left[\Delta_{H 1} e^{i T_{0}}+\Delta_{H 3} e^{3 i T_{0}}\right. \\
& +\Delta_{H 5} e^{5 i T_{0}}+\Delta_{H 7} e^{i T_{0}}+\Delta_{H 2} e^{i T_{0}}-2 i \lambda T_{0} \\
& +\Delta_{H 4} e^{i T_{0}}-4 i \lambda T_{0}+\Delta_{H 6} e^{i T_{0}}-6 i \lambda T_{0} \\
& \left.+\Delta_{H 8} e^{i T_{0}}-8 i \lambda T_{0}+C C\right]
\end{aligned}
$$

where $\Delta_{H i}^{\prime} \mathrm{s}$, are described by $F_{n}=\frac{F}{k_{s}\left(1-\lambda^{2}\right)}$ and $C C$ stands for complex conjugate. For instance, term $\Delta_{H 1}$ is given in Appendix A.

In order to eliminate the secular terms, it is required that:

$$
\Delta_{H 1}=0
$$

To solve Eq. (41), it is assumed that:

$$
A_{H}=\frac{1}{2} a_{H}\left(T_{1}\right) e^{i \phi_{H}\left(T_{1}\right)}
$$

Substituting Eq. (42) into Eq. (41), and separating the real and imaginary parts, leads to:

$$
\begin{aligned}
& \frac{d \phi_{H}}{d T_{1}}=0 \\
& \frac{d a_{H}}{d T_{1}}=-\left(\Lambda_{1} a_{H}+\Lambda_{3} a_{H}^{3}+\Lambda_{5} a_{H}^{5}+\Lambda_{7} a_{H}^{7}+\Lambda_{9} a_{H}^{9}\right)
\end{aligned}
$$

where $\Lambda_{i}^{\prime}$ s are specified by $F_{n}=\frac{F}{k_{s}\left(1-\lambda^{2}\right)}$ and are given in Appendix A.

Hence, $\phi_{H}=\phi_{0}$, which is a constant value, and $a_{H}$, can be determined by Eq. (43b). Because of the small magnitude of system response, terms $a^{5}, a^{7}$ and $a^{9}$ can be neglected, and integration from Eq. (43b) results in:

$$
a_{H}(t)=\sqrt{\frac{a_{0}^{2} \Lambda_{1}}{\left(\Lambda_{1}+\Lambda_{3} a_{0}^{2}\right) e^{2 \varepsilon \Lambda_{1} \omega_{0} t}-\Lambda_{3} a_{0}^{2}}},
$$

where $a_{H}(t)$ is an asymptotic function, such that initial condition, $\left.a_{H}(t)\right|_{t=0}=a_{0}$, is satisfied. Therefore, the first approximation to $u(t)$ is given by:

$$
\begin{aligned}
u(t)= & \sqrt{\frac{a_{0}^{2} \Lambda_{1}}{\left(\Lambda_{1}+\Lambda_{3} a_{0}^{2}\right) e^{2 \varepsilon \Lambda_{1} \omega_{n} t}-\Lambda_{3} a_{0}^{2}}} \cos \left(\omega_{n} t+\phi_{0}\right) \\
& +\frac{F}{m\left(\omega_{n}^{2}-\Omega^{2}\right)} \cos (\Omega t)+O(\varepsilon) .
\end{aligned}
$$

Parameters $\Lambda_{1}, \Lambda_{3}, \Lambda_{5}, \Lambda_{7}$, and $\Lambda_{9}$ in approximate solution for the hard-non-resonant excited system via Eq. (45) are dependent on system characteristics, as well as external force. The steady-state solution (i.e., $t \rightarrow \infty)$ depends on whether $\Lambda_{1}$ is positive or negative. Since $\Lambda_{1}$ is positive, $e^{2 \varepsilon \Lambda_{H 1} \omega_{n} t} \rightarrow \infty$ as $t \rightarrow \infty$, hence, $a_{H} \rightarrow 0$, and the steady-state solution is $u_{s s}=$ $\frac{F}{m\left(\omega_{n}^{2}-\Omega^{2}\right)} \cos (\Omega t)$. As the time increases, the natural response tends towards zero and the force response dominates the natural response. In fact, the natural response fades away after a short time and the steadystate solution consists of the forced response only. Therefore, there is no need to obtain the second order approximation for $u$.

\section{Numerical examples}

The selected values for the numerical examples are given in Tables 1 and 2 for several cases of soft- and hard-excitation analyses, respectively. For all cases, $k_{s}=1000 \frac{\mathrm{N}}{\mathrm{m}}, m=20 \mathrm{~kg}, d=4 \mathrm{~cm}$ and $D=20 \mathrm{~cm}$, $n=1, s=\frac{1}{2}, L=1 \mathrm{~cm}$, and $\Omega=15 \mathrm{rads}^{-1}$ have been selected.

In order to evaluate the proposed analytical solutions for both soft- and- hard excitations, different sets of system parameter effecting $\varepsilon$, have been chosen, so 
Table 1. The selected values and the affected parameters for the soft-excitation analysis with $n=1$, and $s=\frac{1}{2}$.

\begin{tabular}{|c|c|c|c|c|c|c|c|c|c|c|}
\hline \multirow[b]{2}{*}{ Case } & \multicolumn{4}{|c|}{ Selected values } & \multicolumn{4}{|c|}{ Affected parameters } & \multirow[b]{2}{*}{$c_{1}$} & \multirow[b]{2}{*}{$c_{3}$} \\
\hline & $\begin{array}{c}F \\
(\mathrm{~N})\end{array}$ & $\begin{array}{c}\mu \\
(\text { Pa.s })\end{array}$ & $\begin{array}{c}u_{0} \\
(\mathbf{m})\end{array}$ & $\begin{array}{c}\dot{u}_{0} \\
\left(\mathrm{~ms}^{-1}\right)\end{array}$ & $\varepsilon$ & $\hbar$ & $\zeta$ & $\begin{array}{c}c_{\text {linear }} \\
\left(\mathrm{Nsm}^{-1}\right)\end{array}$ & & \\
\hline 1 & 1.5 & 0.0493 & 0.0011 & 0.9 & 0.05 & 0.0123 & 0.025 & 7.08 & 0.5 & 26.04 \\
\hline 2 & 1.5 & 0.147 & 0.0011 & 0.9 & 0.15 & 0.0369 & 0.075 & 21.2 & 0.5 & 26.04 \\
\hline 3 & 1.5 & 0.294 & 0.0011 & 0.9 & 0.3 & 0.0738 & 0.15 & 42.5 & 0.5 & 26.04 \\
\hline 4 & 10 & 0.0493 & 0.0011 & 0.9 & 0.5 & 0.0123 & 0.025 & 7.08 & 0.5 & 26.04 \\
\hline
\end{tabular}

Table 2. The selected values and the affected parameters for the hard-excitation analysis with $n=1$, and $s=\frac{1}{2}$.

\begin{tabular}{|c|c|c|c|c|c|c|c|c|c|c|}
\hline \multirow[b]{2}{*}{ Case } & \multicolumn{4}{|c|}{ Selected values } & \multicolumn{4}{|c|}{ Affected parameters } & \multirow[b]{2}{*}{$\Lambda_{1}$} & \multirow[b]{2}{*}{$\Lambda_{3}$} \\
\hline & $\begin{array}{c}F \\
(\mathbf{N})\end{array}$ & $\begin{array}{c}\mu \\
(\text { Pa.s })\end{array}$ & $\begin{array}{c}u_{0} \\
(\mathrm{~m})\end{array}$ & $\begin{array}{c}\dot{u}_{0} \\
\left(\mathrm{~ms}^{-1}\right)\end{array}$ & $\varepsilon$ & $\hbar$ & $\zeta$ & $\begin{array}{c}c_{\text {linear }} \\
\left(\mathrm{Nsm}^{-1}\right)\end{array}$ & & \\
\hline 1 & 150 & 0.0493 & 0.0001 & 0.75 & 0.05 & 0.0123 & 0.025 & 7.08 & 0.606 & 38.435 \\
\hline 2 & 150 & 0.147 & 0.0001 & 0.75 & 0.15 & 0.0369 & 0.075 & 21.2 & 0.606 & 38.435 \\
\hline 3 & 150 & 0.294 & 0.0001 & 0.75 & 0.3 & 0.0738 & 0.15 & 42.5 & 0.606 & 38.435 \\
\hline
\end{tabular}

that the accuracy of the solutions can be challenged for different values of $\varepsilon$, i.e. different nonlinearity strength yet in weakly categorized nonlinear analysis.

Using these values, parameters $\omega_{n}, \gamma, \beta_{1}, \beta_{2}, \beta_{3}$ and $\beta_{4}$ can be calculated for all cases given in Tables 1 and 2. Those are:

$$
\begin{aligned}
& \omega_{n}=7.071 \mathrm{rads}^{-1}, \quad \gamma=5, \\
& \beta_{1}=208.3, \quad \beta_{2}=16059, \\
& \beta_{3}=542000, \quad \beta_{4}=6782 * 10^{3} .
\end{aligned}
$$

For instance, for the first case of soft-excitation, according to Table $1, \mu=0.0493 \mathrm{~Pa}$.s. The values of the affected parameters are computed as:

$$
\begin{aligned}
& \hbar=0.0123, \quad \varepsilon=0.05, \\
& \zeta=0.025, \quad C_{\text {linear }}=7.08 \mathrm{Nsm}^{-1}, \\
& c_{1}=0.5, \quad c_{3}=26.04,
\end{aligned}
$$

with the initial conditions, given in Table 1 , as: $u(0)=$ $0.0011 \mathrm{~m}, \dot{u}(0)=0.9 \mathrm{~ms}^{-1}$ and $F=1.5 \mathrm{~N}$, along with $\Omega=15 \mathrm{rads}^{-1}$. Since the initial velocity assures the preliminary damping force, initial conditions are considered as: $u(0)=0.0001 \mathrm{~m}$ and $\dot{u}(0)=0.9 \mathrm{~ms}^{-1}$. It is worth mentioning that should the initial velocity be zero, there would be no damping force at the beginning of the movement, which is equivalent to a system with no damper in its mechanism. In addition, the responses of the traditional system and the system with the NDD damper would be similar. Therefore, for better comparison between NDD and the traditional damper, the velocity at the beginning of the movement

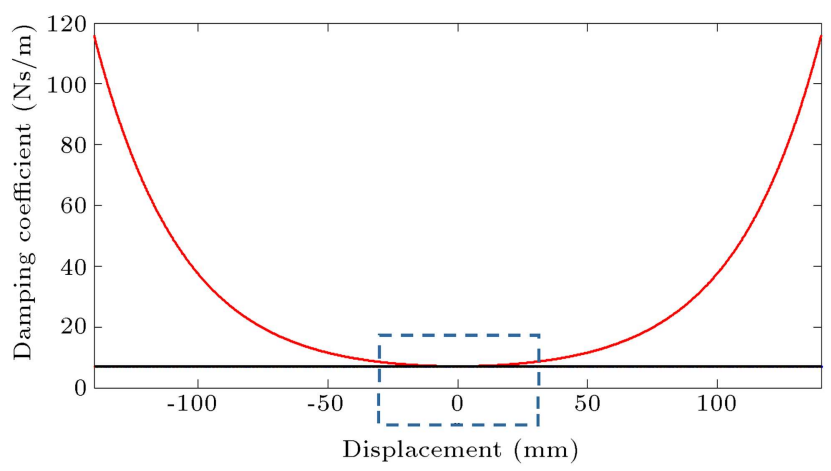

Figure 3. Comparison between the damping coefficients of the NDD damper and the linear damper for $\varepsilon=0.05$. Red line: the NDD damper, and blue line: the linear damper.

in a forced vibration analysis is considered as a nonzero variable.

For more discussion, given that the external force amplitude in soft-excitation analysis must be a small value [35], if the initial velocity is considered as zero, the displacement of the damper becomes insignificant. Therefore, the difference between the damping coefficient of a NDD damper and a traditional linear damper becomes narrow (see the highlighted part in Figure 3 ) and, consequently the performances of two dampers become same.

The transient response amplitude for the soft-nonresonant excited system and the aforementioned initial conditions, is evaluated as follows:

$$
a(t)=0.1269 \sqrt{\frac{1}{0.919 e^{0.3535 t}-0.4193}} .
$$

And, finally, the approximate solution $u(t)$, computed by Eq. (33), for the soft-excitation, is: 


$$
\begin{aligned}
& u(t)=a(t) \cos (7.0700 t-1.155)-4.285 \\
& \times 10^{-4} \cos (15 t)-\left(46551.6 a(t)^{9}+4787.8 a(t)^{7}\right. \\
& \left.+188.9 a(t)^{5}+3.27 a(t)^{3}\right) \sin (21.210 t-3.465) \\
& -\left(1108.3 a(t)^{9}+88.67 a(t)^{7}+2.1 a(t)^{5}\right) \sin (35.350 t \\
& -5.775)-\left(193.9 a(t)^{9}+8.87 a(t)^{7}\right) \sin (49.490 t \\
& -8.085)-16.625 a(t)^{9} \sin (63.630 t-10.395) .
\end{aligned}
$$

For this case of soft-excitation, i.e. the first case of Table 1, the analytical solution of the excited system with the linear damper is:

$$
\begin{aligned}
& u_{l-s}(t)=0.127 e^{-0.177 t} \sin (7.068 t+0.004) \\
& +0.013 * 10^{-4} \sin (15 t) \\
& \quad-4.28 * 10^{-4} \cos (15 t)
\end{aligned}
$$

For the first case of hard-excitation, according to Table 2, $\mu=0.0493$ Pa.s. Using $F=150 \mathrm{~N}$, the values of the affected parameters are computed as:

$$
\begin{array}{ll}
\hbar=0.0123, & \varepsilon=0.05 \\
\zeta=0.025, & C_{\text {linear }}=7.08 \mathrm{Nsm}^{-1}, \\
\Lambda_{1}=0.606, & \Lambda_{3}=38.435 .
\end{array}
$$

The aforementioned initial conditions and external force yield the following relation for $a_{H}(t)$, which exhibits the transient response amplitude for a hardnon-resonant excited system:

$$
a_{H}(t)=0.0908 \sqrt{\frac{1}{1.13 e^{0.429 t}-0.523}} .
$$

Finally, the approximate solution, $u(t)$, calculated by Eq. (45), for the hard-excitation, is:

$$
\begin{aligned}
u(t)= & 0.0908 \sqrt{\frac{1}{1.13 e^{0.429 t}-0.523}} \cos (7.071 t-1.194) \\
& -0.0435 \cos (15 t)
\end{aligned}
$$

For this case of hard-excitation, i.e. the first case of Table 2, the analytical solution of the excited system with the linear damper is specified as:

$$
\begin{aligned}
u_{l-h}(t)= & 0.113 e^{-0.177 t} \sin (7.068 t+0.39) \\
& +0.0013 \sin (15 t)-0.0428 \cos (15 t)
\end{aligned}
$$

\section{Results and discussion}

The damping coefficients of a NDD damper and a traditional linear damper versus displacement are shown in Figure 3 for the first case of Tables 1 and 2 with $\varepsilon=0.05$. As can be seen in Figure 3, the damping coefficient of the traditional damper is a constant value (that is, $7.13 \mathrm{~N} . \mathrm{s} / \mathrm{m}$ ), whereas the damping coefficient of a NDD damper changes as the system moves. This mainly explains the difference between a system equipped with a NDD damper and a system with a traditional linear damper.

The simulation results for the soft-non-resonant excitation analysis are shown in Figures 4-7. In order to verify the accuracy of the soft-excitation response of

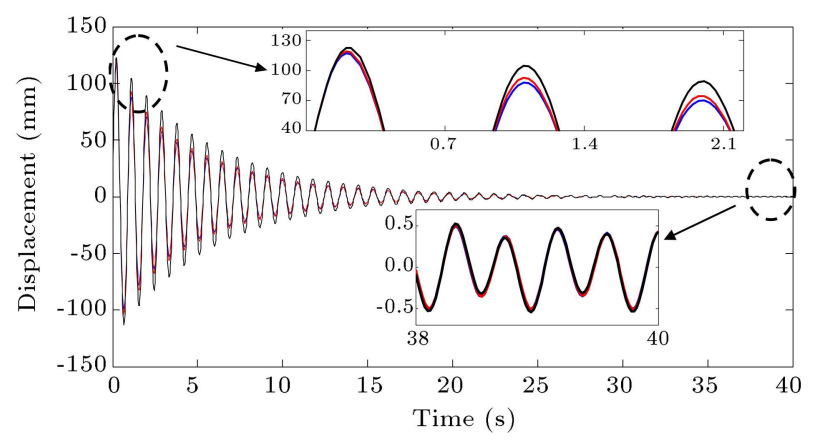

(a)

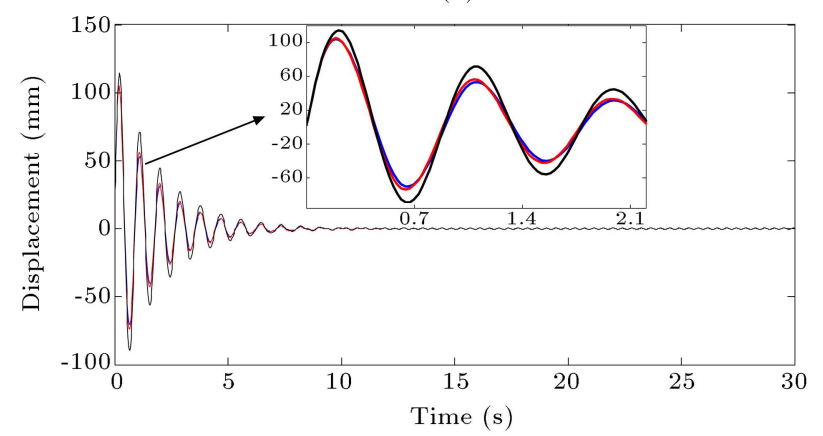

(b)

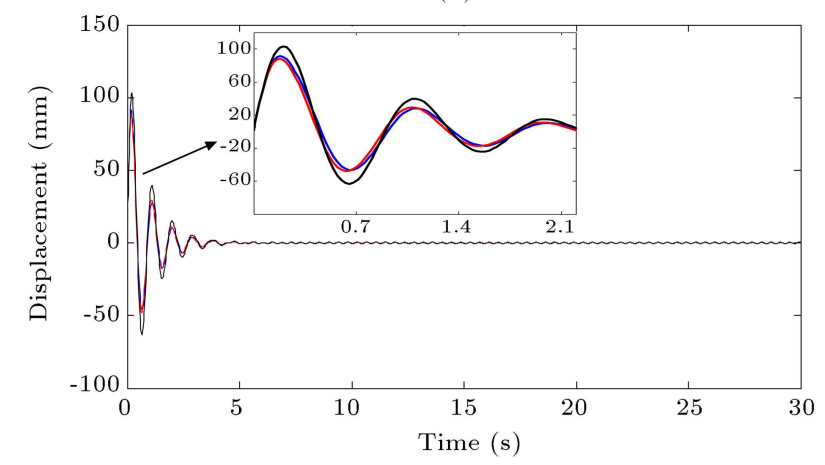

(c)

Figure 4. Response of the soft-excited system with $F=1.5 \mathrm{~N}$ for (a) $\varepsilon=0.05$, (b) $\varepsilon=0.15$, (c) $\varepsilon=0.30$. Red line: approximate analytical solution of the system with the NDD damper; blue line: numerical solution of the system with the NDD damper; and black line: analytical solution of the system with the linear damper. 


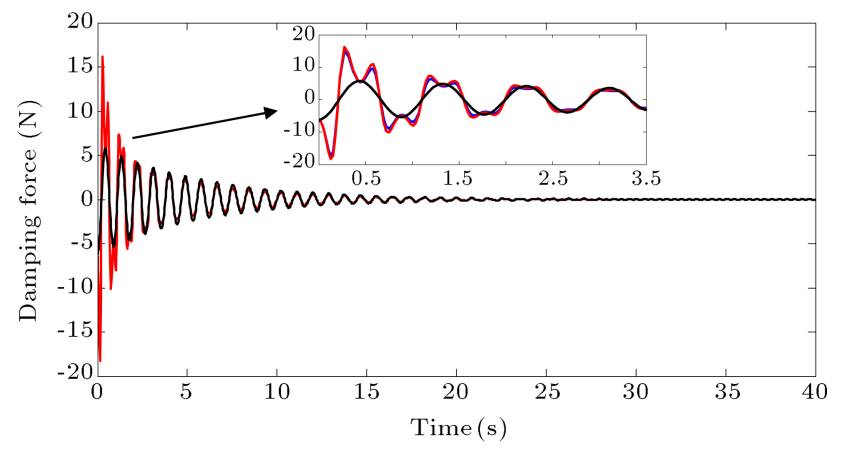

Figure 5. Damping force of the soft-excited system with $F=1.5 \mathrm{~N}$ for $\varepsilon=0.05$. Red line: damping force of the NDD damper obtained by the MSM; blue line: damping force of the NDD damper obtained by the Runge-Kutta method; and black line: damping force of the linear damper.

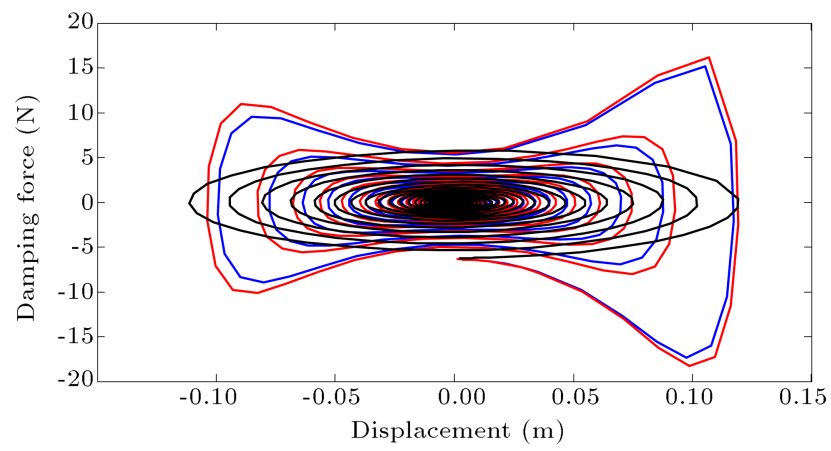

Figure 6. Damping force versus displacement of the soft-excited system with $F=1.5 \mathrm{~N}$ for $\varepsilon=0.05$. Red line: damping force of the NDD damper obtained by the MSM; blue line: damping force of the NDD damper obtained by the Runge-Kutta method; and black line: damping force of the linear damper.

the system equipped with the NDD damper, i.e. the solution of Eq. (33), the numerical integration method is also applied to solving Eq. (11).

Figure 4(a) shows the comparison between the approximate analytical solution Eq. (33) and the numerical result obtained by the Runge-Kutta method for the first case given in Table 1 , with $F=1.5 \mathrm{~N}$ and $\varepsilon=0.05$. It can be seen that the approximate analytical solution is nearly identical to the result obtained by the Runge-Kutta method (see insets of Figure 4(a)). This comparison is carried out for cases 2 and 3 from Table 1 . The results are illustrated in Figures 4(b) and (c) for different values of $\varepsilon$. The analytical solution of the system with the traditional linear damper is also depicted in Figure 4 for cases 1,2 and 3 from Table 1. As shown in Figure 4, as the value of $\varepsilon$ rises, the error between the approximate analytical solution (Eq. (33)) and the exact numerical solution of Eq. (11) grows. This is due to the properties of the multiple scales method, whereas the value of $\varepsilon$ must be small. Thus, the approximate analytical solutions are

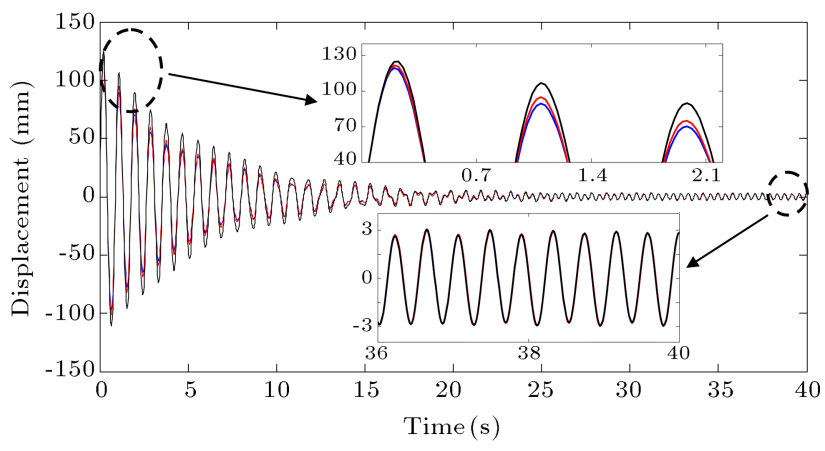

Figure 7. Response of the soft-excited system with $F=10 \mathrm{~N}$ and $\varepsilon=0.05$. Red line: approximate analytical solution of the system with the NDD damper; blue line: numerical solution of the system with the NDD damper; and black line: analytical solution of the system with the linear damper.

satisfactory for each case. According to Figure 4, using the NDD damper causes more reduction in vibration amplitude for the soft-excited system compared to the system with the linear damper. The amplitude of the system with the NDD damper is reduced in all cycles until the response of the system tends to the steady-state solution. For example, the amount of vibration amplitude reduction for case 1 from Table 1 , with $F=1.5 \mathrm{~N}$ and $\varepsilon=0.05$, is $4.6 \%$ in the first cycle, $19 \%$ in the second cycle, $27.32 \%$ in the third cycle, $32.54 \%$ in the fourth cycle, $36.2 \%$ in the fifth cycle, and $38.39 \%$ in the sixth cycle. For this case, as shown enlarged in Figure 4(a), the responses of the soft-excited system with the NDD damper, as well as the linear damper, tend to the steady-state solution as $u_{s s}(t)=-4.282 * 10^{-4} \cos (15 t-0.03)$, after about 38 seconds. Due to the fact that raising the value of $\varepsilon$, and, hence, strengthening nonlinearity causes the natural response to tend to zero after a shorter time, and the soft-excitation response to tend more rapidly towards its steady-state solution. Therefore, the percentages of amplitude reduction for the first three cycles in all cases are presented in Table 3 . Table 3 clearly demonstrates the NDD damper performance in reducing the amplitude of the softexcited system.

The damping forces versus time for the system equipped with the NDD damper are shown in Figure 5

Table 3. The percentage of amplitude reduction in each cycle for the soft-excitation analysis.

\begin{tabular}{ccccc}
\hline \multirow{2}{*}{ Case } & \multirow{\varepsilon}{*}{} & \multicolumn{3}{c}{ Cycle } \\
\cline { 3 - 5 } & & $\mathbf{1}$ & $\mathbf{2}$ & $\mathbf{3}$ \\
\hline 1 & 0.05 & 4.6 & 19 & 27.32 \\
2 & 0.15 & 10 & 34 & 41.7 \\
3 & 0.30 & 12.97 & 41.4 & 45.7 \\
4 & 0.05 & 4.77 & 19.2 & 28 \\
\hline
\end{tabular}


for the first case in Table 1. The damping force of the linear damper is also depicted in Figure 5 for that case. As mentioned previously, based on Figure 3, the damping coefficient of the NDD damper changes as the system moves. As a result, the higher displacement of the system leads to a higher damping coefficient for the NDD damper, and, consequently, a higher damping force, whilst, the damping coefficient of a linear damper is constant during the movement of the system. Thus, the NDD damper in the first cycles, in which the displacement is significantly large, produces more effective force in the opposite direction of the movement compared to the traditional linear damper (see inset Figure 5). This causes more amplitude reduction of the vibration compared to the linear damper (see Figure 4).

The damping forces versus displacement for the system equipped with the NDD damper are shown in Figure 6, for the first case, in Table 1. The curve of damping force versus displacement exhibits the work done by the damper that is the amount of energy absorbed by the damper. According to Figure 6, the NDD damper provides more energy dissipation than the linear damper in the soft-excited system, particularly in the first cycles of vibration.

To study the effect of increasing the amount of external force on the response of the soft-excitation, for case 1 , given in Table 1 , with $F=1.5$, the amplitude of the external force is increased to $F=10 \mathrm{~N}$, as case 4 , in Table 1. The simulation result is shown in Figure 7. It is observed that for this case, the steady-state solution not only has higher amplitude, but also the amount of vibration amplitude is more reduced compared to case 1 . According to Figure 7, the vibration amplitude reduction is $4.77 \%$ in the first cycle, $19.2 \%$ in the second cycle, $28 \%$ in the third cycle, $34.58 \%$ in the fourth cycle, $39.14 \%$ in the fifth cycle, and $40.27 \%$ in the sixth cycle.

The simulation results for the hard-non-resonant excitation analysis are shown in Figures 8-10. Figure 8 shows the comparison between the approximate analytical solution and the numerical result obtained by the Runge-Kutta method for all cases of Table 2, with $F=150 \mathrm{~N}$, and different values of $\varepsilon$. It can be seen that the approximate analytical solutions are nearly identical to the results obtained by the RungeKutta method in all hard-excitation cases. As shown in Figure 8 , as the value of $\varepsilon$ rises, the error between the approximate analytical solution (Eq. (45)) and the exact numerical solution of Eq. (11) grows. According to Figure 8, using the NDD damper causes more reduction in the vibration amplitude for the hard-excited system than when utilizing the linear damper. Also, the amplitude of the system with the NDD damper compared to the system with the linear damper is reduced in all cycles until the response of the system tends to

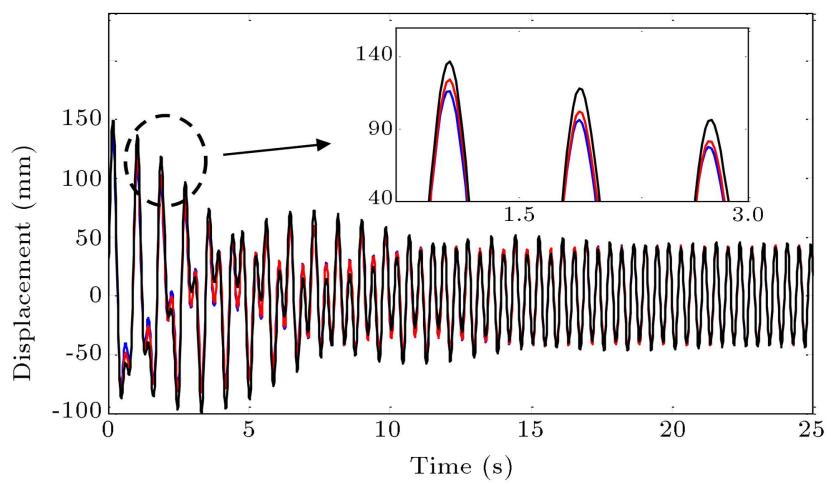

(a)

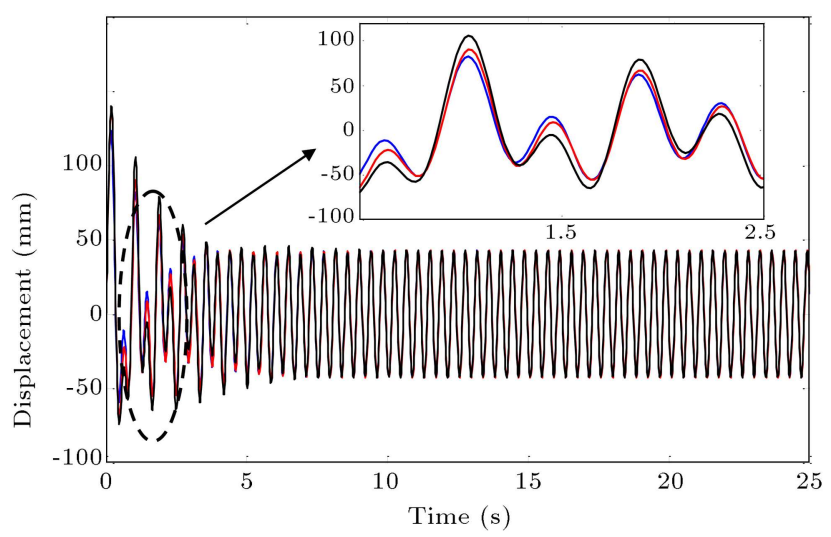

(b)

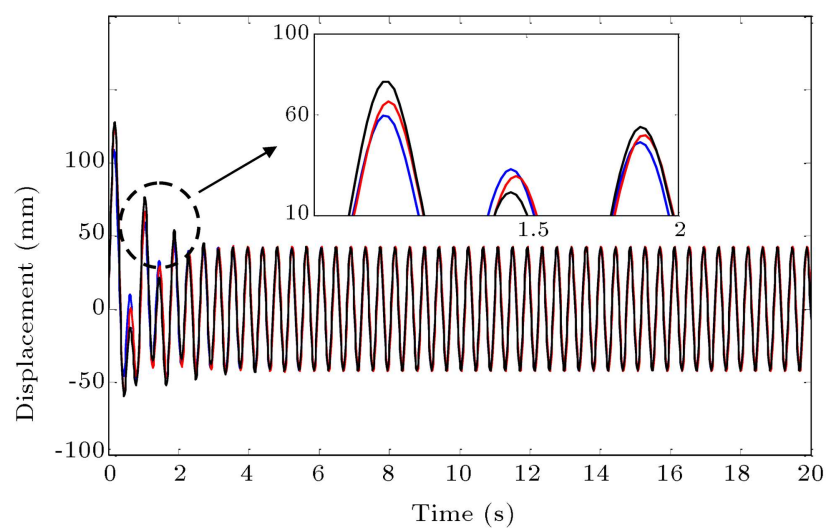

(c)

Figure 8. Response of the hard-excited system with $F=150 \mathrm{~N}$ for (a) $\varepsilon=0.05$, (b) $\varepsilon=0.15$, and (c) $\varepsilon=0.30$. Red line: approximate analytical solution of the system with the NDD damper; blue line: numerical solution of the system with the NDD damper; and black line: analytical solution of the system with the linear damper.

the steady-state solution, as $u_{s s}=\frac{F}{m\left(\omega_{n}^{2}-\Omega^{2}\right)} \cos (\Omega t)$. The percentages of amplitude reduction for the first three cycles in all hard-excitation cases are presented in Table 4 . Table 4 also clearly demonstrates the NDD damper performance in reducing the amplitude of the hard-excited system.

The damping forces versus time for the system equipped with the NDD damper for case 1 given in 


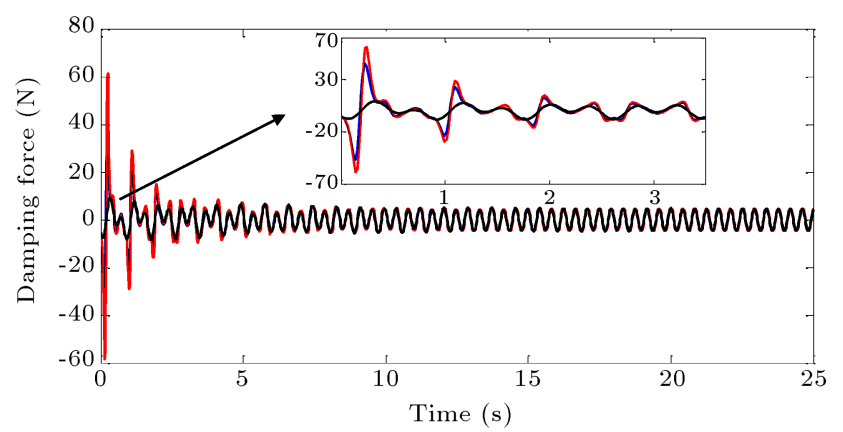

Figure 9. Damping force of the hard-excited system with $F=150 \mathrm{~N}$ for $\varepsilon=0.05$. Red line: damping force of the NDD damper obtained by the MSM; blue line: damping force of the NDD damper obtained by the Runge-Kutta method; and black line: damping force of the linear damper.

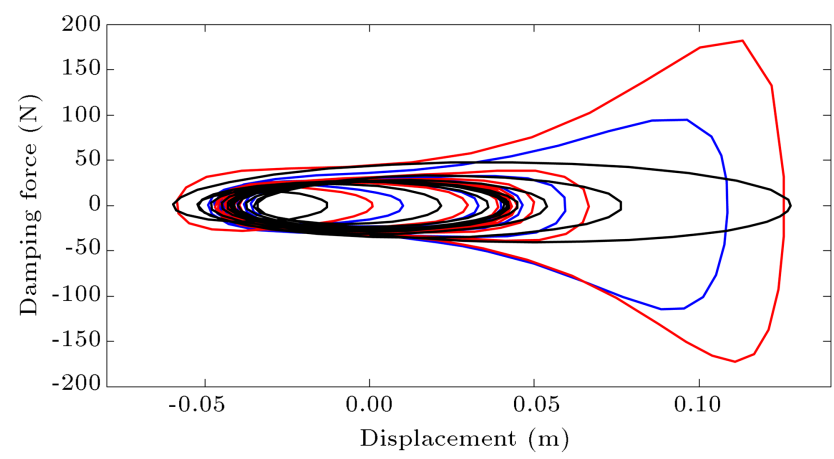

Figure 10. Damping force versus displacement of the hard-excited system with $F=150 \mathrm{~N}$ for $\varepsilon=0.05$. Red line: damping force of the NDD damper obtained by the MSM; blue line: damping force of the NDD damper obtained by the Runge-Kutta method; and black line: damping force of the linear damper.

Table 4. The percentage of amplitude reduction in each cycle for the hard-excitation analysis.

\begin{tabular}{ccccc}
\hline \multirow{2}{*}{ Case } & \multirow{\varepsilon}{*}{$\boldsymbol{c}$} & \multicolumn{3}{c}{ Cycle } \\
\cline { 3 - 5 } & & $\mathbf{1}$ & $\mathbf{2}$ & $\mathbf{3}$ \\
\hline 1 & 0.05 & 6.58 & 17.89 & 22.59 \\
2 & 0.15 & 13.11 & 28.4 & 27.09 \\
3 & 0.30 & 17.66 & 28.32 & 15.94 \\
\hline
\end{tabular}

Table 2 is shown in Figure 9. The damping force using the linear damper is also depicted in Figure 9 for that case of hard-excitation. Due to the property of the NDD damper illustrated in Figure 3, the NDD damper in the first cycles, where the displacement is significantly large, creates more effective force in the opposite direction of movement compared to the traditional linear damper (see insets of Figure 9).

The damping forces versus displacement for the system equipped with the NDD damper is also shown in Figure 10 for the first case of hard-excitation given in Table 2. According to Figure 10, the NDD damper pro-

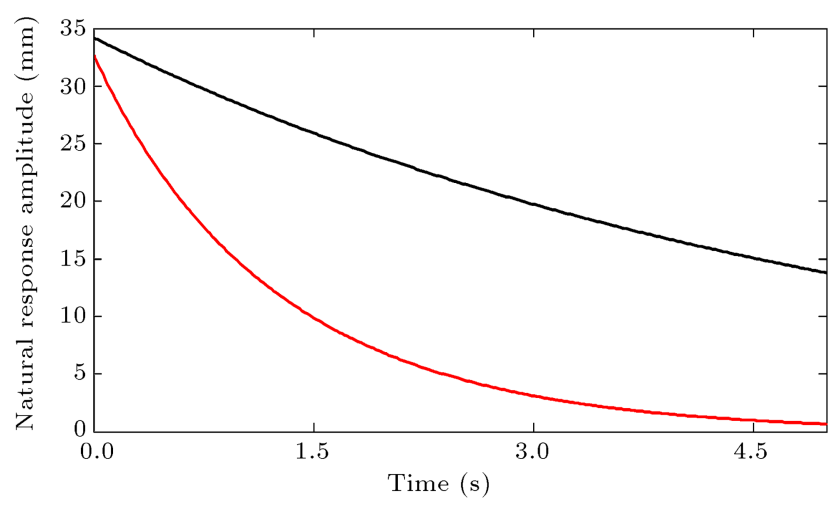

Figure 11. Natural response amplitude profile of the excited mass-spring-NDD damper system with $\varepsilon=0.05$ and $F=420$ N. Purple line: $a(t)$ in the soft-excitation algorithm; and red line: $a_{H}(t)$ in the hard-excitation algorithm.

vides more energy dissipation than the linear damper in the hard-excited system.

In order to compare the responses obtained by soft-excitation and hard-excitation algorithm, the following example is presented.

Consider the amplitude of the external force to be extremely large, as $F=420 \mathrm{~N}$, with the initial condition: $u_{0}=-0.14 \mathrm{~mm}$ and $\dot{u}_{0}=0.2 \mathrm{~ms}^{-1}$ for the value of $\varepsilon=0.05$.

Figure 11 illustrates the comparison between $a(t)$ obtained from Eq. (32), where the forced vibration has been classified as soft-excitation, and $a_{H}(t)$ obtained from Eq. (44), in which the forced vibration has been considered hard-excitation. Due to parameters $\Lambda_{1}$ and $\Lambda_{3}$, stated as Eq. (A.7) in Appendix A, the natural response amplitude in the hard-excitation algorithm, i.e. $a_{H}(t)$, is affected by the external force via Eq. (44). As the excitation amplitude rises, the values of $\Lambda_{1}$ and $\Lambda_{3}$, and, subsequently, $e^{\varepsilon \Lambda_{1} \omega_{0} t}$, are increased. Therefore, $a_{H}(t)$ and the natural response tend to zero in a short time, as shown in Figure 11. In fact, in the hard-excitation algorithm, the force response dominates the natural response by tending to the steady-state solution. In the soft-excitation algorithm, however, parameters $c_{i}$ used in Eq. (26b) are fixed and force-independent values, which result in $a(t)$ becoming independent of the external force also. Therefore, in spite of large excitation amplitude, $a(t)$ and the natural response tend to zero after a long time (see Figure 11). Figure 12 demonstrates a comparison between the final solutions acquired by the soft-excitation algorithm via Eq. (33) and the hard-excitation algorithm via Eq. (45). The numerical integration using the fourthorder Rung Kutta method is also carried out in order to compare the accuracy of the approximate solutions. For the large amplitude of external excitation, i.e. $F=$ $420 \mathrm{~N}$, in contrast to the solution obtained by the softexcitation algorithm, the approximate analytical solu- 


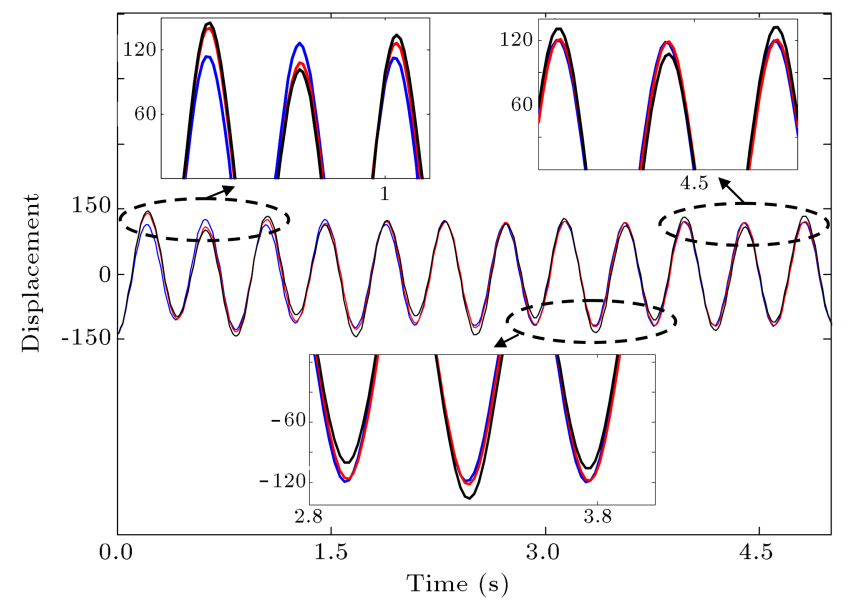

Figure 12. Response of the excited mass-spring-NDD damper system with $\varepsilon=0.05$ and $F=420 \mathrm{~N}$ obtained by: Purple line: the soft-excitation algorithm; red line: the hard-excitation algorithm; and blue line: the Runge-Kutta method.

tion obtained by the hard-excitation algorithm follows more precisely the numerical solution computed by the Runge-Kutta method (see enlarged insets of Figure 12). In fact, in the proposed hard-excitation algorithm, the natural response of the system is considerably modified by $a_{H}(t)$ shown in Figure 11, and results in a more accurate response compared to the soft-excitation solution.

\section{Conclusion}

Forced vibration analysis of a simple mass-spring system equipped with a Nonlinear DisplacementDependent (NDD) damper is studied in this paper. The approximate analytical solution algorithms were developed by the method of multiple scales for two types of soft- and hard-excitation. The advised hard- and soft-excitation algorithms were performed for several cases and their accuracy was also verified by the numerical fourth-order Runge-Kutta method. The results confirm that for both soft- and hardexcitations, the multiple scales method is able to achieve the satisfactory response performance. Also, utilizing the NDD damper in the soft- or hard-excited system causes more vibration amplitude reduction than in the traditional linear damper. Furthermore, it was found that increasing the strength of nonlinearity of the system, which is related to the parameter $\varepsilon$, results in the natural response tending to zero after a shorter time, and the response of the system tends rapidly to its steady-state solution. In addition, for the hard-excitation analysis with considerable external force amplitude, the response of the system tends to its steady-state solution in a shorter time compared to the soft-excited system with the same nonlinearity. Therefore, organizing the external force based on its amplitude into soft- and hard-excitation, results in a better estimated response in the forced vibration analysis. In particular, for large amplitude of the external excitation force, the hard-excitation solution yields more accurate results than the soft-excitation solution.

\section{References}

1. De Silva, C.W., Vibration: Fundamentals and Practice, 2nd Ed. CRC Press, Boca Raton (2006).

2. Rao, S.S., Mechanical Vibrations, 4th Ed., PrenticeHall, Englewood Cliffs (2004).

3. Tomson, W.T. and Diloon Dahleh, M., Theory of Vibration with Applications, 5th Ed. Prentice-Hall (1998).

4. Dixon, J.C., The Shock Absorber Handbook, 2nd Ed. Wiley, Chichester (2007)

5. Eslaminasab, N. "Development of a semi-active intelligent suspension system for heavy vehicles", Ph.D. Thesis, University of Waterloo, Canada (2008).

6. Zhou, N. and Liu, K. "A tunable high-static-lowdynamic stiffness vibration isolator", J. Sound Vib., 329, pp. 1254-1273 (2010).

7. Cetin, S., Zergeroglu, E., Sivirioglu, S. and Yuksek, I. "A new semiactive nonlinear adaptive controller for structures using MR damper: design and experimental vibration", Nonlinear Dyn., 66(4), pp. 731-743 (2011)

8. Dong, X.-M., Yu, M., Liao, C.-R. and Chen, W.-M. "Comparative research on semi-active control strategies for msgnrt-rheological suspension", Nonlinear Dyn., 59, pp. 433-453 (2010).

9. Song, X. and Ahmadian, M. "Characterization of semi-active control system dynamics with magnetorheological suspensions", J. Vib. Control, 16(10), pp. 1439-1463 (2010)

10. Go, C.-G., Sui, H., Shih, M.-H. and Sung, W.-P. "A linearization model for the displacement dependent semi-active hydraulic damper", J. Vib. Control, 16(14), pp. 219-2214 (2010).

11. Preumont, A., Vibration Control of Active Structures, Kluwer Academic Publishers Dordrecht, EBook ISBN: 0-306-48422-6 (2002).

12. Crosby, M.J. and Karnopp, D.C. "The active damper: a new concept for shock and vibration control", Shock and Vibrations Bulletin, 43, pp. 119-133 (1973).

13. Ahmadian, M. "On the isolation properties of semiactive dampers", Journal of Vibration and Control, 5(2), pp. 217-232 (1999).

14. Simon, D.E. "Experimental evaluation of semiactive magneto-rheological primary suspensions for heavy truck applications", PhD Thesis, Virginia Tech (2000).

15. Venkatesan, C. and Krishnan, R. "Harmonic response of a shock mount employing dual-phase damping", Journal of Sound and Vibration, 40(3), pp. 409-413 (1975). 
16. Jackson, G.W. "Fundamentals of the direct acting shock absorber", SAE paper 37R, National Passenger Car Body and Materials Meeting, Detroit (1959).

17. Puydak, R.C. and Auda, R.S. "Designing to achieve optimum dynamic properties in elastomeric cab and body mounts", SAE 660439 (and SAE Transactions V75) (1966).

18. Lewitske, C. and Lee, P. "Application of elastomeric components for noise and vibration isolation in the automotive industry", SAE Paper 2001-01-1447 (2001).

19. Fukushima, N., Hidaka, K. and Iwata, K. "Optimum characteristics of automotive shock absorbers under various driving conditions and road surfaces", JSAE Review, pp. 62-69 (1983).

20. Young, D.W. "Aircraft landing gears", Proc. I. Mech. E., 200(D2), pp. 75-92 (1986).

21. Etman, L.F.P., Vermeulen, R.C.N., Van Heck, J.G.A.M., Schoofs, A.J.G. and Van Campen, D.H. "Design of a stroke dependent damper for the front axle suspension of a truck using multibody system dynamics and numerical optimization", Vehicle System Dyn., 38, pp. 85-101 (2002)

22. Komamura, S. and Mizumukai, K. "History of shock absorbers", Automobile Technology, 41(1), pp. 126-131 (1987) (in Chinese).

23. Lee, C.-T. and Moon, B.-Y. "Study on the damping performance characteristics analysis of shock absorber of vehicle by considering fluid force", Journal of $\mathrm{Me}$ chanical Science and Technology, 19(2), pp. 520-528 (2005).

24. Lee, C.-T. and Moon, B.-Y. "Simulation and experimental validation of vehicle dynamic characteristics for displacement-sensitive shock absorber using fluid-flow modeling", Mechanical Systems and Signal Processing, 20, pp. 373-388 (2006)

25. Hundal, M.S. "Impact absorber with two-stage variable area orifice hydraulic damper", Journal of Sound and Vibration, 50(2), pp. 195-202 (1977).

26. Haque, M.M., Ahmed, A.K.W. and Sankar, S. "Simulation of displacement sensitive non-linear dampers via integral formulation of damping force characterization", Journal of Sound and Vibration, 187, pp. 95-109 (1995).

27. Farjoud, A., Ahmiadian, M., Craft, M. and Burke, W. "Nonlinear modeling and experimental characterization of hydraulic dampers: effects of shim stack and orifice parameters on damper performance", Nonlinear Dyn., 67(2), pp. 1437-1456 (2012).

28. Guo, P.F., Lang, Z.Q. and Peng, ZK. "Analysis and design of the force and displacement transmissibility of nonlinear viscous damper based vibration isolation systems", Nonlinear Dyn., 67(4), pp. 2671-2687 (2012).

29. Ilbeigi, Sh., Jahanpour, J. and Farshidianfar, A. "A novel scheme for nonlinear displacement-dependent dampers", Nonlinear Dyn., 70, pp. 421-434 (2012).
30. Sung, W.P. and Shih, M.H. "Testing and modeling for energy dissipation behavior of velocity and displacement dependent hydraulic damper", Tsinghua Science \& Technology, 13, pp. 1-6 (2008).

31. Bugra, H., Ertas, B.H. and Luo, H. "Nonlinear dynamic characterization of oil-free wire mesh dampers", Gas Turbines Power, 130(3), 032503 (2008).

32. Main, J.A. and Jones, N.P. "Analysis and design of linear and nonlinear dampers for stay cables", Proceedings Fourth International Symposium on Cable Dynamics, Montreal, Canada, AIM (2001).

33. Diotallevi, P.P., Landi, L. and Dellavalle, A. "Simplified design methodology for systems equipped with non-linear viscous dampers", The 14th World Conference on Earthquake Engineering, Beijing, China (2008).

34. Nayfeh, A.H. and Mook, D.T., Nonlinear Oscillations, Wiley, New York (1979).

35. Nayfeh, A.H., Perturbation Methods, Wiley, New York (1973).

\section{Appendix A}

The parameters defined in Eqs. (11), (21), (25), (28b), (33), (40) and (43b) are as follows:

$$
\begin{aligned}
\beta_{1}= & \frac{\left(4 \gamma^{4}-4 \gamma^{2}\right) \beta}{\left(\gamma^{4}-2 \gamma^{2}+1\right)}, \\
\beta_{2}= & \frac{\left(6 \gamma^{4}-2 \gamma^{2}\right) \beta^{2}}{\left(\gamma^{4}-2 \gamma^{2}+1\right)}, \\
\beta_{3}= & \frac{4 \gamma^{4} \beta^{3}}{\left(\gamma^{4}-2 \gamma^{2}+1\right)}, \\
\beta_{4}= & \frac{\gamma^{4} \beta^{4}}{\left(\gamma^{4}-2 \gamma^{2}+1\right)}, \\
\Delta_{1}= & \beta_{1} A^{2} \bar{A}+5 \beta_{3} A^{4} \bar{A}^{3}+2 \beta_{2} A^{3} \bar{A}^{2}+14 \beta_{4} A^{5} \bar{A}^{4} \\
& +A+2 \partial A / \partial T_{1}, \\
\Delta_{2}= & \beta_{1} A^{3}+3 \beta_{2} A^{4} \bar{A}+9 \beta_{3} A^{5} \bar{A}^{2}+28 \beta_{4} A^{6} \bar{A}^{3}, \\
\Delta_{3}= & \beta_{2} A^{5}+5 \beta_{3} A^{6} \bar{A}+20 \beta_{4} A^{7} \bar{A}^{2}, \\
\Delta_{4}= & \beta_{3} A^{7}+7 \beta_{4} A^{8} \bar{A}, \\
\Delta_{5}= & \beta_{4} A^{9}, \quad c_{9}=\frac{7}{256} \beta_{4}, \\
c_{1}= & 0.5, \quad c_{3}=0.125 \beta_{1}, \quad c_{5}=0.625 \beta_{2}, \\
c_{7}= & \frac{10}{256} \beta_{3}, \quad(\mathrm{~A} .2)
\end{aligned}
$$




$$
\begin{aligned}
& q\left(T_{1}, T_{2}\right)=\frac{1}{8} \beta_{1}^{2} A^{3} \bar{A}^{2}+\beta_{1} \beta_{2} A^{4} \bar{A}^{3}+\frac{23}{12} \beta_{2}^{2} A^{5} \bar{A}^{4} \\
& +\frac{15}{4} \beta_{1} \beta_{3} A^{5} \bar{A}^{4}+14 \beta_{2} \beta_{3} A^{6} \bar{A}^{5} \\
& +14 \beta_{1} \beta_{4} A^{6} \bar{A}^{5}+\frac{1205}{48} \beta_{3}^{2} A^{7} \bar{A}^{6} \\
& +\frac{154}{3} \beta_{2} \beta_{4} A^{7} \bar{A}^{6}+\frac{545}{3} \beta_{3} \beta_{4} A^{8} \bar{A}^{7} \\
& +\frac{13013}{40} \beta_{4}^{2} A^{9} \bar{A}^{8} \\
& \Upsilon_{1}=\frac{1}{8} \beta_{1} a^{3}+\frac{3}{32} \beta_{2} a^{5}+\frac{9}{128} \beta_{3} a^{7}+\frac{7}{128} \beta_{4} a^{9}, \\
& \Upsilon_{2}=\frac{1}{32} \beta_{2} a^{5}+\frac{5}{128} \beta_{3} a^{7}+\frac{5}{128} \beta_{3} a^{7}, \\
& \Upsilon_{3}=\frac{1}{128} \beta_{3} a^{7}+\frac{7}{512} \beta_{4} a^{9} \\
& \Upsilon_{4}=\frac{1}{512} \beta_{4} a^{9} \\
& \Delta_{H 1}=\left(\frac{35}{128} \beta_{4} A+70 \beta_{4} A^{4} \bar{A}^{3}\right)\left(F_{n}\right)^{8} \\
& +\left(\frac{5}{16} \beta_{3} A+\frac{35}{4} \beta_{4} A^{2} \bar{A}\right)\left(F_{n}\right)^{6} \\
& +\left(\frac{3}{8} \beta_{2} A+\frac{45}{8} \beta_{3} A^{2} \bar{A}+\frac{105}{2} \beta_{4} A^{3} \bar{A}^{2}\right)\left(F_{n}\right)^{4} \\
& +\left(\frac{1}{2} \beta_{1} A+3 \beta_{2} A^{2} \bar{A}+15 \beta_{3} A^{3} \bar{A}^{2}\right)\left(F_{n}\right)^{2} \\
& +\left(A+2 \partial A / \partial T_{1}+\beta_{1} A^{2} \bar{A}+2 \beta_{2} A^{3} \bar{A}^{2}\right. \\
& \left.+5 \beta_{3} A^{4} \bar{A}^{3}+14 \beta_{4} A^{5} \bar{A}^{4}\right)\left(F_{n}\right)^{0}, \\
& \Lambda_{1}=\frac{35}{256} \beta_{4}\left(F_{n}\right)^{8}+\frac{5}{32} \beta_{3}\left(F_{n}\right)^{6}+\frac{3}{16} \beta_{2}\left(F_{n}\right)^{4} \\
& +\frac{1}{4} \beta_{1}\left(F_{n}\right)^{2}+\frac{1}{2}\left(F_{n}\right)^{0}
\end{aligned}
$$

$$
\begin{aligned}
& \Lambda_{3}=\frac{35}{32} \beta_{4}\left(F_{n}\right)^{6}+\frac{45}{64} \beta_{3}\left(F_{n}\right)^{4}+\frac{3}{8} \beta_{2}\left(F_{n}\right)^{2}+\frac{1}{8} \beta_{1}\left(F_{n}\right)^{0}, \\
& \Lambda_{5}=\frac{105}{64} \beta_{4}\left(F_{n}\right)^{4}+\frac{15}{32} \beta_{3}\left(F_{n}\right)^{2}+\frac{1}{16} \beta_{2}\left(F_{n}\right)^{0}, \\
& \Lambda_{7}=\frac{35}{64} \beta_{4}\left(F_{n}\right)^{2}+\frac{5}{128} \beta_{3}\left(F_{n}\right)^{0}, \\
& \Lambda_{9}=\frac{7}{256} \beta_{4}\left(F_{n}\right)^{0} .
\end{aligned}
$$

\section{Biographies}

Javad Jahanpour received his BS degree in Mechanical Engineering from Ferdowsi University of Mashhad (FUM), Iran, his MS degree in Applied Mechanical Engineering from Isfahan University of Technology (IUT), Isfahan, Iran, and a PhD degree with first class Honours in Mechanical Engineering, in 2008, from Ferdowsi University of Mashhad (FUM), Iran. From 2008 to 2009, he was a post-doctoral researcher at National Cheng Kung University (NCKU), Taiwan. He is currently Assistant Professor in the Mechanical Engineering Department at the Islamic Azad University of Mashhad (IAUM), Iran. His research interests include trajectory generation and control motion problem with applications in robotics, vibration and CAD/CAM systems.

Mojtaba Porghoveh received his BS degree in Mechanical Engineering, in 2011, from the Islamic Azad University of Mashhad (IAUM), Iran, where he is currently an MS degree student in Applied Mechanics.

Shahab Ilbeigi received his BS degree in Mechanical Engineering from the Islamic Azad University of Mashhad (IAUM), Iran, in 2008, and an MS degree in Applied Mechanical Engineering from Bu-Ali Sina University, Hamedan, Iran, in 2012. He is currently a $\mathrm{PhD}$ degree student in the Department of Mechanical, Industrial and Systems Engineering at the University of Rhode Island, Kingston, USA. His research interests include nonlinear modeling/analysis, CAD/CAM, and optimization problems. 